\title{
Perioperative Adjuvant Therapy
}

National Cancer Institute

\section{Source}

National Cancer Institute. Perioperative Adjuvant Therapy. NCI Thesaurus. Code C16031.

Treatment given at the time of an operation to augment the effects of the operation. 